\title{
Current Trends in Peritoneal Dialysis
}

\section{Kunal Chaudhary ${ }^{1,2 *}$}

${ }^{1}$ Truman VA Hospital, Columbia, MO, USA

${ }^{2}$ Division of Nephrology, University of Missouri, Columbia, MO, USA

\section{Introduction}

Starting 2011, CMS decided that there will be one payment for dialysis treatments regardless of whether the treatment is $\mathrm{PD}$ or $\mathrm{HD}$, and whether performed at home or in a dialysis center. PD is the least expensive form of dialysis therapy due to several reasons. The supplies for PD are less expensive and it is less labor intensive for nurses; the patient and family providing the majority of labor. Also, fewer parenteral medications are given to $\mathrm{PD}$ patients than to $\mathrm{HD}$ patients, given the more convenient oral route of administration and the lack of a permanent intravenous access. Third, significantly lesser and lower dosages of ESAs are given to PD patients due to a more efficient ESA response(likely due to increased marrow response and subcutaneous dosing), preserved residual renal function, and less blood loss (no obligate HD loss). However, under the previous payment structure the larger "separately billables" portion of billing for injectable drugs given at HD served as a profit for dialysis providers, who took advantage of the spread between acquisition costs and payment rate. The percentage of profits of some dialysis chains solely from the use of ESAs was a staggering $20 \%$ of the total profits. This provider dependence upon separately billable services had perhaps impeded the use of less costly $\mathrm{PD}$, accounting for its decline as a renal replacement therapy in the USA $[1,2]$.

\section{$\mathrm{PD}$ as a viable option}

The recent inclusion of injectables within the bundled singlepayment strategy is a strong incentive for the dialysis provider to encourage home PD. By 2014 other oral medications such as phosphate binders and calcimimetics will be included in the single payment bundle. Under the bundled payment for dialysis services, greater utilization of PD is expected to result in greater profitability. The rationale is that expensive intravenous medications will be contained within the single bundle payment and, as PD patients require fewer intravenous medications, this savings would make delivery of PD care less costly to the provider. The current FDA recommendation for ESRD patients with anemia is to start ESAs when the Hb level is below $10 \mathrm{~g} / \mathrm{dL}$ and to discontinue or decrease the ESA dose when the $\mathrm{Hb}$ level is above $11 \mathrm{~g} / \mathrm{dL}$, these targets are achieved relatively easily in PD patients with a combination of s.c ESA and oral iron preparations. Many dialysis providers anticipated even greater ESA cost savings following the implementation of bundling in 2011 through the use of lower $\mathrm{Hb}$ targets, greater use of IV iron and administration of ESAs via the subcutaneous (SC) rather than the intravenous (IV) route. The effects of the bundling are already becoming evident and the DOPPS database has reported a nationwide decrease in haemoglobin levels since the June 2011 ESA label update. The mean haemoglobin level has declined by $0.10 \mathrm{~g} / \mathrm{dL}$ from August 2010 to July 2011 and then by an additional $0.29 \mathrm{~g} / \mathrm{dL}$ from July to October 2011, before appearing to stabilize. In December 2011, the mean Hgb level was $11.09 \mathrm{~g} / \mathrm{dL}$ (10.94 g/dL in ESA-treated patients). From August 2010 through December 2011, the percentage of patients with haemoglobin greater than $12 \mathrm{~g} /$ $\mathrm{dL}$ declined from $32 \%$ to $22 \%$.

Besides the economic benefits under the bundled payment system there are other reasons to consider PD as a "first" dialysis modality
[3]. Along with improved survival, other long-term goals of ESRD patients are to improve quality of life, preserve residual renal function, and reduce morbidity. Infection-related complications are higher and appear to be increasing in HD patients, whereas such complications have steadily declined in PD patients over the last few years. Earlier studies had shown a survival advantage in the first 1-2 years of PD vs. HD which disappeared in later years, however more recent studies have shown that there was no difference in 5 year survival in the cohorts starting after 2002, and the risk of death and technique failure of PD was less in the recent cohorts [4,5]. Patient satisfaction seems to be better amongst PD patients as compared those on $\mathrm{HD}$, and $\mathrm{PD}$ patients are more likely to rate the dialysis care they receive as excellent compared to HD [6]

\section{Conclusion}

Thus besides similar outcomes of PD compared to HD, the economic incentives of decreasing use of injectables, most notably ESAs, lesser staffing requirements, equipment needs, space requirements, and facility overhead, providers could administer more PD care with less utilization of resources and take advantage of the inherent profitability of PD under the new bundle. A survey of top 10 dialysis providers in the US has shown an increase from $7.6 \%$ to $8.2 \%$ of PD population from May 2010 to May 2011, a trend which is likely to continue in the for seeable future.

\section{References}

1. Bethesda MD (2009) USRDS data system: USRDS annual data report.

2. Mehrotra R, Kermah D, Fried L, Khawar O, Norris K, et al. (2007) Chronic peritoneal dialysis in the United States: declining utilization despite improving outcomes. J Am Soc Nephrol 18: 2781-2788.

3. Chaudhary K, Sangha H, Khanna R (2011) Peritoneal dialysis first: rationale Clin J Am Soc Nephrol 6: 447-56.

4. Mehrotra R, Chiu YW, Kalantar-Zadeh K, Bargman J, Vonesh E (2011) Similar outcomes with hemodialysis and peritoneal dialysis in patients with end-stage renal disease. Arch Intern Med 171: 110-118

5. McDonald SP, Marshall MR, Johnson DW, Polkinghorne KR (2009) Relationship between dialysis modality and mortality. J Am Soc Nephrol 20: 155-163

6. Rubin HR, Fink NE, Plantinga LC, Sadler JH, Kliger AS, et al. (2004) Patient ratings of dialysis care with peritoneal dialysis vs hemodialysis. JAMA 291: 697-703.

${ }^{*}$ Corresponding author: Kunal Chaudhary MD, FACP, FASN, Associate Professo of Clinical Medicine, Division of Nephrology, University of Missouri Health Center 1 Hospital drive, CE 422, Columbia, MO 65212, USA, E-mail: chaudharyk@health. missouri.edu

Received June 21, 2012; Accepted June 24, 2012; Published June 26, 2012

Citation: Chaudhary K (2012) Current Trends in Peritoneal Dialysis. J Nephrol Therapeut 2:e107. doi:10.4172/2161-0959.1000e107

Copyright: @ 2012 Chaudhary K. This is an open-access article distributed under the terms of the Creative Commons Attribution License, which permits unrestricted use, distribution, and reproduction in any medium, provided the original author and source are credited. 